\title{
Serum total thiol level in Acne Vulgaris Patients and its relation to oxidative stress N.W.Mikhael ${ }^{1}$, S.M.Rezk ${ }^{1}$, H.A.Abdel Khalik ${ }^{2}$ and M.A.Mowafy ${ }^{1}$ \\ ${ }^{1}$ Dermatology, Venerology and Andrology, Dept., Faculty of Medicine, Benha Univ., Benha, Egypt \\ ${ }^{2}$ Clinical and Chemical Pathology, Dept., Faculty of Medicine, Benha Univ., Benha, Egypt \\ Email:
}

\begin{abstract}
Background:Acne vulgaris (AV): is the most frequent dermatologic illness and is the chronic, multifactoral, inflammatory disease of the pilosebaceous unit. Aim: to compare the dynamic balance between thiol/disulfide and the balance of healthy individuals in AV sufferers. Methods: This research has been performed on 40 individuals who have been clinically diagnosed with AV. Furthermore, 40 seemingly healthy people of matching sex and age are controlled. Each patient had a thorough history, a comprehensive clinical examination and a laboratory study for: Total Thiol and thiol disulphide serum level. Results \& Findings: The age varied from 16 to 31 years with mean \pm SD $=$ $(22.40 \pm 4.35)$ years, while the control age was between 16 and 31 years with mean $\pm \mathrm{SD}=(23.83 \pm 4.99)$ years, with no significant statistical differences $(\mathrm{p}=0.288)$ between both groups. This age was considered to have been between 16 and 31 years. Comparative for serum total thiol, serum thiol disulfide and their ratio between the two groups examined. The serum total thiol $(\mathrm{p}<0,001)$ and serum thiol disulfide $(\mathrm{p}<0,001)$ in the AV group were significantly increased as compared to the control group. Although the total thiol/thiol disulfide ratio $(\mathrm{p}<0.001)$ in $\mathrm{AV}$ group has decreased considerably compared with the control group.
\end{abstract}

Keywords: Dynamic, Thiol, Disulfide, Balance, Acne Vulgaris.

\section{Introduction}

Acne vulgaris (AV) is the most prevalent dermatological disorder of the chronic, complex, inflammatory pilosebean unit, affecting 80 percent in the population 11-30 years [1].

There are many variables that play a part in AV development, the major causes of acnes, inflammation and immunological response, sebum hyperplasia and colonisation of Propionibacterium acnes, play a leading role in disease pathogenesis [2].

Thiols are chemical molecules containing sulphur, which form complexes and are necessary for the biological system with amino acids and proteins. Thiols are essential antioxidants that play a vital role in the elimination via enzyme and non-enzyme mechanisms of reactive oxygen species. The Thiol plasma pool is of low molecular weight. Thiols include cysteine, homocysteine, glutathione and albumin - and protein thiol in particular. Thiols undergo an oxidation process forms a disulfide bond with oxidant molecules. For detoxification, the control of pathways of signalling, and apoptosis and enzyme activities dynamic thiol/disulfide homeostasis (TDH) is indispensable[3].

Studies have shown that the aetiology of several hair disorders, such as psoriasis, is linked with dysregulated TDH. In telogenetic effluvium occurs, for example, various conditions such as diabetes, cardiovascular illnesses, malignant tumours and Parkinson's disease[4] [5].

The research aimed at comparing the dynamic thiol/disulfide balance with that of healthy individuals in patients with $\mathrm{AV}$.

\section{Patients and methods}

This was a case-control study, was conducted on 30 patients suffering from AV with various acne severity levels (Patient group) and 23 healthy individuals of matched age and sex as a (control group). Patients were recruited from the outpatient clinic of Dermatology and Andrology Department of Banha University Hospitals between 0ctober2020 and January 2021.

The local ethic committee approved the study of Banha Faculty of Medicine. Informed consent was obtained from each individual before enrollement in the study.

\subsection{Inclusion Criteria}

- Patients more than or equal 15 years old with different degrees of AV severity.

- Patients hadn't taken any medications for acne for one month before taking samples.

\subsection{Exclusion Criteria}

Patients excluded from the study were those:

- Suffering from chronic inflammatory disease.

- Drinking alcohol or tobacco smoking.

- Pregnant or lactating female.

- Suffering of Diabetes mellitus, cancers, and hypertension.

- Taking any medication or oral contraceptive pills in the last four weeks before the study.

Each patient was subjected to full history taking, complete clinical examination, and Laboratory investigation for: Serum level of Total Thiol and Thiol Disulphide by human total thiol Elisa Kit:( Cat No:E02463hu.Langham Creek )and by human thiol disulfide Elisa Kit: (Cat NoE-02415hu.Langham Creek).

\subsection{Statistical analysis}

Data was collected, coded then entered as a spread sheet using Microsoft Excel 2016 for Windows, Microsoft Corporation, United States. Data was 
analyzed using IBM Statistical Package for Social Sciences software (SPSS), 21st edition, IBM, United States. The Kolmogorov-Smirnov test was used to verify the normality of distribution. Continuous data was expressed as mean \pm standard deviation, median \& IQR while categorical data as numbers and percentage. Data was presented as tables and graphs. Results was considered statistically significant at a pvalue of less than or equal 0.05 and highly statistically significant at a p-value of less than or equal 0.001 . The used tests were

- Chi-square test For categorical variables, to compare between different groups

- Fisher's Exact test Correction for chi-square when more than $20 \%$ of the cells have expected count less than 5

- Student T-test For normally distributed quantitative variables, to compare between two studied groups

- Mann Whitney test For abnormally distributed quantitative variables, to compare between two studied groups

\begin{abstract}
3. Results
This study was conducted on 40 patients Clinically diagnosed AV patient. In addition ,40 apparently healthy individuals of matched age and sex as a control group. This study was conducted on outpatient clinic of Dermatology, Andrology and Venereology department of Benha university hospitals. The age in AV patients group ranged from 16 to 31 years with mean $\pm \mathrm{SD}=(22.40 \pm 4.35)$ years while the in control group the age ranged from 16 to 31 years with mean $\pm \mathrm{SD}=(23.83 \pm 4.99)$ years with no statistical significant difference $(\mathrm{p}=0.288)$ between the two groups. There was no statistical significant difference between the two groups as regards gender, weight, height and BMI ( $\mathrm{p}=0.698,0.548,0.472$ and 0.723 respectively). Also, there was no statistical significant difference between the two groups as regards occupation, menstruation and marital status $(\mathrm{p}=0.577,0.554$ and 0.949 respectively) table (1).
\end{abstract}

Table (1) Demographic characteristics among the two studied groups.

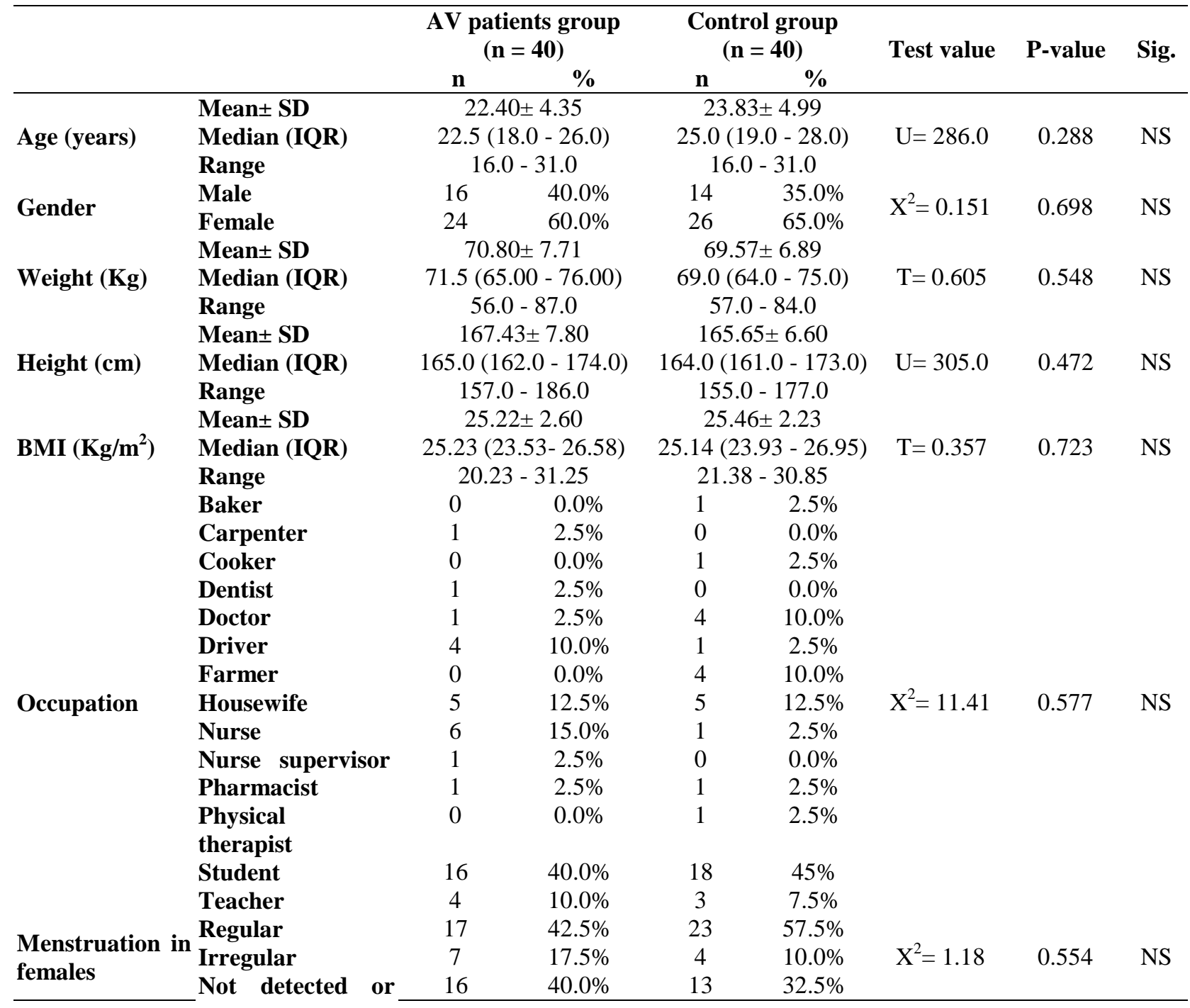




\begin{tabular}{|c|c|c|c|c|c|c|c|c|}
\hline & & \multicolumn{2}{|c|}{$\begin{array}{l}\text { AV patients group } \\
(n=40)\end{array}$} & \multicolumn{2}{|c|}{$\begin{array}{c}\text { Control group } \\
(n=40)\end{array}$} & \multirow[t]{2}{*}{ Test value } & \multirow[t]{2}{*}{ P-value } & \multirow[t]{2}{*}{ Sig. } \\
\hline & & $\mathbf{n}$ & $\%$ & $\mathbf{n}$ & $\%$ & & & \\
\hline Marital status & $\begin{array}{l}\text { male } \\
\text { Single } \\
\text { Married }\end{array}$ & $\begin{array}{l}17 \\
23 .\end{array}$ & $\begin{array}{l}42.5 \% \\
57.5 \%\end{array}$ & $\begin{array}{l}16 \\
24\end{array}$ & $\begin{array}{l}40.0 \% \\
60.0 \%\end{array}$ & $X^{2}=0.004$ & 0.949 & NS \\
\hline
\end{tabular}

$\mathrm{p} \leq 0.05$ is considered statistically significant, $\mathrm{p} \leq 0.01$ is considered high statistically significant,

$\mathrm{SD}=$ standard deviation, -comparison between groups done by Independent Samples Student T test, Chi-Square Test and Mann- Whitney U test

Table (2) Clinical characteristics in AV patients group.

\begin{tabular}{|c|c|c|c|}
\hline & & \multicolumn{2}{|c|}{$\begin{array}{l}\text { AV patients group } \\
(n=40)\end{array}$} \\
\hline & & $\mathbf{n}$ & $\%$ \\
\hline \multirow{3}{*}{ Age of onset of AV (years) } & Mean \pm SD & \multicolumn{2}{|c|}{$17.57 \pm 1.72$} \\
\hline & Median (IQR) & \multicolumn{2}{|c|}{$17.0(16.0-19.0)$} \\
\hline & Range & \multicolumn{2}{|c|}{$15.0-20.0$} \\
\hline \multirow{4}{*}{ Duration of disease (years) } & Mean \pm SD & \multicolumn{2}{|c|}{$4.93 \pm 3.27$} \\
\hline & Median & \multicolumn{2}{|c|}{$4.5(2.0-7.0)$} \\
\hline & Range & \multicolumn{2}{|c|}{$1.0-11.0$} \\
\hline & Face & 12 & $30.0 \%$ \\
\hline \multirow{3}{*}{ Distribution } & Face \& Chest & 9 & $22.5 \%$ \\
\hline & Face \&Back & 3 & $7.5 \%$ \\
\hline & Face\& Back\& Chest & 16 & $40.0 \%$ \\
\hline \multirow{2}{*}{ Course of disease } & Intermittent & 17 & $42.5 \%$ \\
\hline & Progressive & 23 & $57.5 \%$ \\
\hline \multirow{2}{*}{ PIH } & No & 19 & $47.5 \%$ \\
\hline & Yes & 21 & $52.5 \%$ \\
\hline \multirow{2}{*}{ Scar formation } & No & 21 & $52.5 \%$ \\
\hline & Yes & 19 & $47.5 \%$ \\
\hline \multirow{4}{*}{ Scar type } & icepick & 10 & $25 \%$ \\
\hline & Rolling & 5 & $12.5 \%$ \\
\hline & boxcar & 3 & $7.5 \%$ \\
\hline & hypertrophic & 1 & $2.5 \%$ \\
\hline \multirow{3}{*}{ GAGS score } & Mean \pm SD & \multicolumn{2}{|c|}{$24.93 \pm 6.55$} \\
\hline & Median (IQR) & \multicolumn{2}{|c|}{$24.0(21.0-27.0)$} \\
\hline & Range & \multicolumn{2}{|c|}{$13.0-37.0$} \\
\hline \multirow{3}{*}{$\begin{array}{l}\text { Severity according to GAGS } \\
\text { score }\end{array}$} & Mild & 5 & $12.5 \%$ \\
\hline & Moderate & 25 & $62.5 \%$ \\
\hline & Severe & 10 & $25 \%$ \\
\hline
\end{tabular}

$\mathrm{SD}=$ standard deviation, -comparison between groups done by Pearson Chi Square test and Mann- Whitney U test

Table (2) shows clinical characteristics in AV patients group. The mean $( \pm$ SD) age of onset of AV was $17.57( \pm 1.72)$ years while the mean $( \pm$ SD) duration in those patients was $4.93( \pm 3.27)$ years. The majority of cases had AV in face, back and chest (40\%). Post-inflammatory hyperpigmentation (PIH) was found in $52.5 \%$ of cases and formation of scar was detected in $47.5 \%$ of the cases. $50 \%$ patients with scar had icepick type (fig. 9). GAGS score ranged from 13 to 37 with mean $( \pm$ SD) $24.93( \pm 6.55)$. Most patients
(62.5\%) had moderate degree of acne according to GAGS score.

On comparison between the two studied groups as regard serum total thiol, serum thiol disulfide and their ratio. There was high significant increase in serum total thiol $(p<0.001)$ and serum thiol disulfide $(\mathrm{p}<0.001)$ in $\mathrm{AV}$ group compared to control group (fig. 1-2). While there was high significant decrease in total thiol/thiol disulfide ratio $(\mathrm{p}<0.001)$ in $\mathrm{AV}$ group compared to control group fig. (3). 


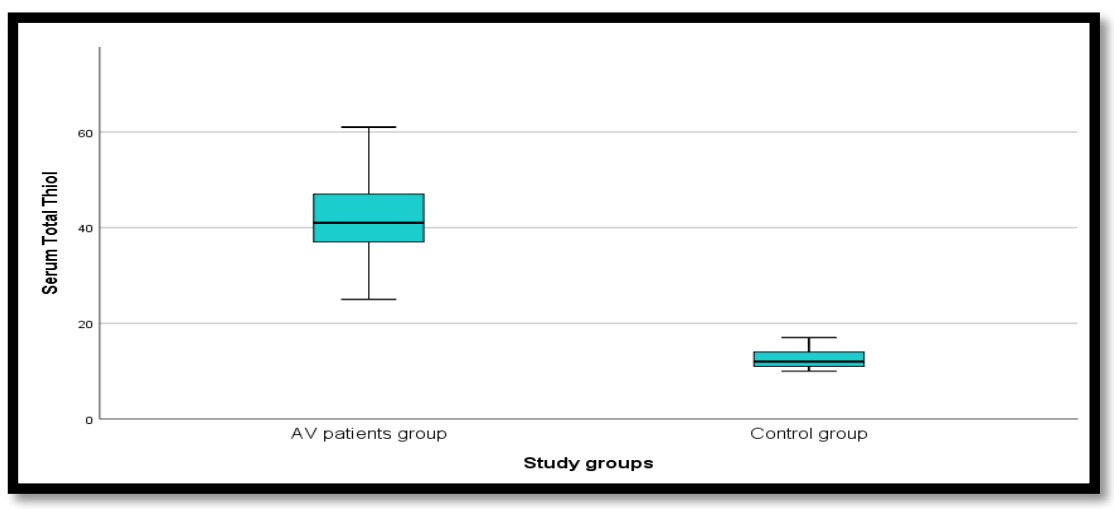

Fig. (1) Boxplot showing comparison between AV patients group and control group regarding serum total thiol.

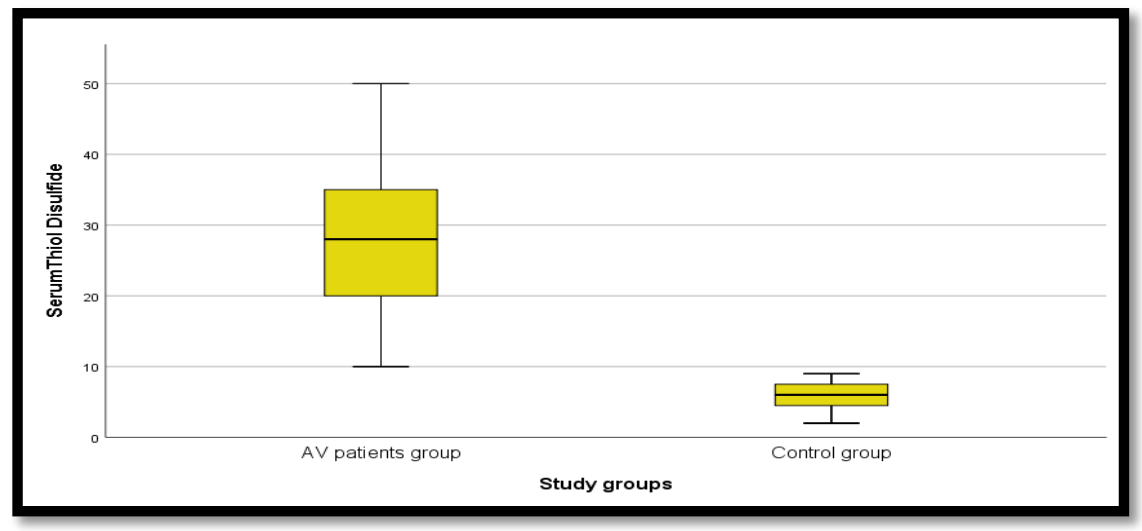

Fig. (2) Boxplot showing comparison between AV patients group and control group regarding serum thiol disulfide.

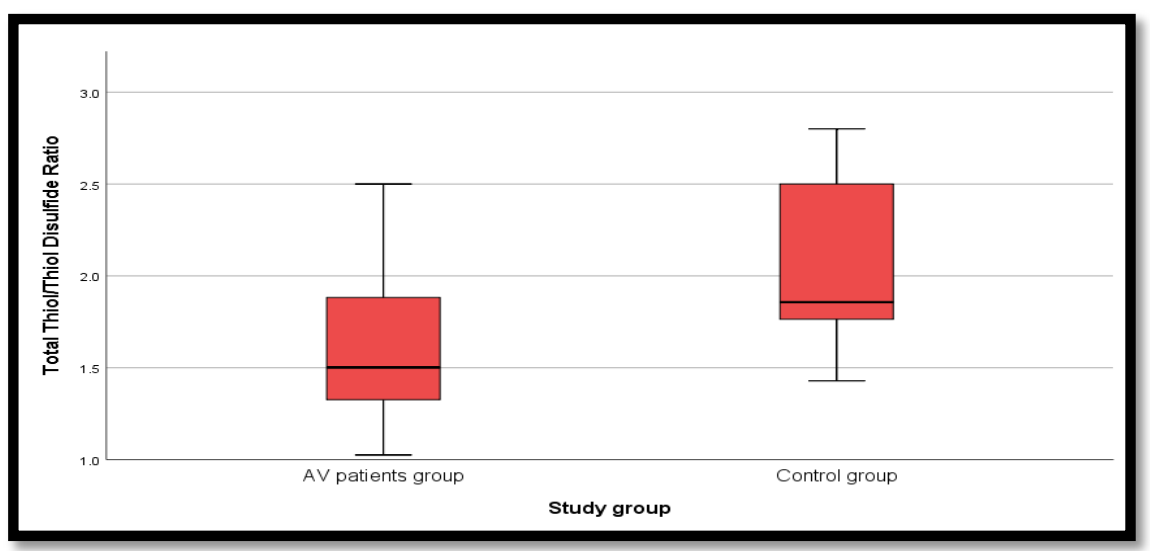

Fig. (3) Boxplot showing comparison between AV patients group and control group regarding total thiol/ thiol disulfide ratio.

Table (3) shows correlations analysis between total thiol/thiol disulfide ratio and other parameters in the studied groups. In AV patients, a significant negative correlation was found between total thiol/thiol disulfide ratio with AGA $(r=-0.374$, $\mathrm{p}=0.042)$, serum thiol disulfide $(\mathrm{r}=-0.86, \mathrm{p}<0.001)$ and serum total thiol $(\mathrm{r}=-0.581, \mathrm{p}=0.001)$. While, there was significant positive correlation was found between total thiol/thiol disulfide ratio with BMI ( $r=$ 0.455, $\mathrm{p}=0.01)$, scar formation $(\mathrm{r}=0.54, \mathrm{p}=0.002)$,
GAG score $(\mathrm{r}=0.626, \mathrm{p}<0.001)$, stress and fatigue $(\mathrm{r}=$ $0.424, \mathrm{p}=0.020)$ and serum thiol disulfide $(\mathrm{r}=0.871$, $\mathrm{p}<0.001)$. In control group, it was noticed that there was positive correlation between total thiol/thiol disulfide ratio with weight $(\mathrm{r}=0.488, \mathrm{p}=0.018)$, height $(\mathrm{r}=0.457, \mathrm{p}=0.028)$ and menstruation $(\mathrm{r}=0.500, \mathrm{p}=$ $0.015)$. while, a significant negative correlation was found between total thiol/thiol disulfide ratio with gender $(r=-0.566, p=0.005)$, eating nuts $(r=-0.621$, $\mathrm{p}=0.002)$ and serum thiol disulfide $(\mathrm{r}=-0.77, \mathrm{p}<0.001)$. 
Table (3) Correlations analysis between total thiol/thiol disulfide ratio and other parameters in the studied groups.

\begin{tabular}{|c|c|c|c|c|}
\hline & \multicolumn{4}{|c|}{$\begin{array}{c}\text { Total Thiol/Thiol Disulfide Ratio } \\
\end{array}$} \\
\hline & \multicolumn{2}{|c|}{$\begin{array}{l}\text { AV patients group } \\
(n=40)\end{array}$} & \multicolumn{2}{|c|}{$\begin{array}{c}\text { Control group } \\
(\mathbf{n}=\mathbf{4 0})\end{array}$} \\
\hline & $\mathbf{r}$ & p-value & $\mathbf{r}$ & p- value \\
\hline Age & -0.028 & 0.882 & 0.000 & 0.999 \\
\hline BMI & 0.455 & 0.011 & 0.096 & 0.664 \\
\hline Age of disease onset & 0.124 & 0.515 & - & - \\
\hline Course of disease (progressive) & 0.031 & 0.870 & - & - \\
\hline Duration of disease & -0.183 & 0.333 & - & - \\
\hline PIH (+ve) & 0.228 & 0.226 & - & - \\
\hline Scar formation (+ve) & 0.540 & 0.002 & - & - \\
\hline GAG score & 0.626 & $<0.001$ & - & - \\
\hline Association with AGA(+ve) & -0.374 & 0.042 & -0.008 & 0.971 \\
\hline Facial cleanser (frequency of use) & 0.064 & 0.737 & -0.129 & 0.559 \\
\hline Sunblock (frequency of use) & 0.063 & .0741 & -0.053 & 0.810 \\
\hline Physical exercise (frequency) & 0.088 & 0.642 & 0.286 & 0.185 \\
\hline Sleep hours & -0.147 & 0.438 & -0.030 & 0.891 \\
\hline Stress and fatigue (+ve) & 0.424 & 0.020 & -0.043 & 0.846 \\
\hline Use of Scalp Oil / Bleaching cream & 0.252 & 0.180 & -0.146 & 0.506 \\
\hline Cosmetics use & 0.127 & 0.505 & -0.402 & 0.057 \\
\hline Milk Drinking & 0.126 & 0.508 & -0.382 & 0.072 \\
\hline Dairy products (frequency of use) & 0.230 & 0.221 & -0.241 & 0.269 \\
\hline Fruits (frequency of use) & 0.203 & 0.283 & -0.322 & 0.134 \\
\hline Vegetables (frequency of use) & -0.028 & 0.882 & 0.275 & 0.203 \\
\hline Sea food (frequency of use) & 0.146 & 0.529 & - & - \\
\hline Chocolate (frequency of use) & 0.064 & 0.735 & -0.086 & 0.697 \\
\hline Nuts (frequency of use) & -0.086 & 0.650 & -0.621 & 0.002 \\
\hline Oily food (frequency of use) & -0.054 & 0.776 & -0.385 & 0.070 \\
\hline Serum Total Thiol & -0.581 & 0.001 & -0.022 & 0.922 \\
\hline Serum Thiol Disulfide & -0.860 & $<0.001$ & -0.770 & $<0.001$ \\
\hline
\end{tabular}

$\mathrm{p} \leq 0.05$ is considered statistically significant, $\mathrm{p} \leq 0.01$ is considered highly significant

\section{Discussion}

The present research has shown that serum total thiol and serum thiol disulfide $(\mathrm{p}<0.001)$ have increased significantly in the AV group in comparison with the control group. Although the total thiol/thiol disulfide ratio was significantly changed to thiol disulfide $(p<0.001)$ in AV group compared to the control group.

According to the present results, this major shift to thioldisulphide was observed in many dermatological disorders in the overall ratio of thiol/thiol-disulfide.

Emre et al. observed that in the blood of patients with active SD, the amount of thiol groups is greater than in healthy people, An increase in thiol levels in blood may be related to homeostasis in order to decrease the increasing burden of oxidative stress in individuals with SD, and higher thiol groups may lead to an enhanced proliferation of SD lesions[6].

In addition, Pektas et al., [7] showed in an alopecia areata (AA) research that oxidant stress and its impact on thiol/thiol total disulfide may be caused by widespread hair follicle loss in AA disease. In their research, the group of AA found that the amount of disulphide and the disulphide-total thiol ratio (shift to thiol-disulphide side) were much higher $(\mathrm{p}<0.01)$. It was observed that the overall levels of thiol between the AA and control groups were comparable ( $p>0.05)$, the homeostasis of thiol/disulphide switched to oxidative stress, a reduction in thiols and rise in AA patients.

Aksoy et al. have shown [8], according to current research findings, that the balance of thiol/disulfide was changed to disulphide in psoriasis patients. This is essential to substantiate the involvement of oxidative stress in psoriasis aetiology.

Sener et al.[9] studies indicate that the thioldisulfide balance in rosacea patients was seen in order to move towards disulfides, an indication of oxidative strain in the rosacea. The ROS produced by the variables that cause rosacea was believed to lead to disulfide bonds and thiol levels being reduced by oxidising thiol groups in the cell. Thiol/disulfide homeostasis may be considered as a biomarker reflecting the oxidative stress in rosacea.

Unlike the present findings of the research, Gürel et al.[10] have shown that the disulfide/total thiol proportion in $\mathrm{AV}$ patients is much lower than the control group $(\mathrm{p}=0.034)$ (shifting to total thiol side). Thiol levels and thiol-disulphide levels were shown to vary significantly between patients with AV and control individuals. The authors interpreted these 
inverted results that the oxidative stress associated with AV may come from a disulfide-independent mechanism.

A substantial negative association was observed between total serum thiol and GAG score $(r=-0.676$, $\mathrm{p}<0.001)$ in the present research. A substantial positive connection between the thiol/thiol-disulfide total serum score ratio was observed with GAG $(\mathrm{r}=0.626$, $\mathrm{p}<0.001)$. There was a substantial negative association between serum thiol- disulfide $(\mathrm{r}=0.708, \mathrm{p}<0.001)$ and GAG score.

In accordance with the present research, in the study by Gürel et al.,[10], a GAG score of mild $(n=25)$, moderate $(n=24)$ and serious $(n=25)$ was divided into three sub-groups. Total plasma concentrations of thiol and disulfide were not substantially different across subgroups and not significantly different between individuals with mild, moderate and severe disorders. The authors stated that oxidative stress associated with AV may be caused by an independent mechanism of disulfide levels, which means that there was no connection in the trial between the severity of A.V and total thiol/thioldisulfide ratio.

The recent findings revealed a strong positive connection of serum total thiol with AGA $(r=0.463$, $\mathrm{p}=0.01$ ) in both AV and control groups.

Significant positive connection was observed with the AGA relationship $(\mathrm{r}=0.447, \mathrm{p}=0.013)$ between serum thiol disulfide. The total thiol/thiol disulfides ratio with AGA was shown to be significantly unfavourable in AV patients. $(r=-0.374, p=0.042)$.

Prie et al. have discovered that total antioxidant activity has reduced in patients with AGA in line with current findings, therefore showing oxidative stress in such individuals. Declining significant superoxide dismutase activity (SOD), but no change in catalase, glutathione peroxidase, non-protein thiol levels and the overall antioxidant activity in erythrocytes are elements that suggest the presence of oxidative stress in these patients and a compensatory mechanical mechanism for SOD dysfunction of androgenetic alopecia patients in red blood cells.

There is also a strong positive link between the total thiol/thiol disulfide ratio and $\mathrm{BMI}(\mathrm{r}=0.455, \mathrm{p}=$ 0.011 ) as shown in current studies.

According to the results, Söğüt et al.[12] showed that oxidative stress in groups with a BMI higher than normal is elevated (talk25).

While the overall Thiol/thiol disulfide ratio with scar development was significantly favourable $(r=0.54$, $\mathrm{p}=0.002$ ).

Another research confirms the findings of recent studies by indicating that free radicals play a significant role in skin fibrotic diseases such as scleroderma, GVHD and hypertrophic scars[13]. Other writers were similarly supportive of the function of oxidative stress in skin scarring[14].

\section{Conclusion}

This research found that the total blood thiol and serum thiol-disulfide $(\mathrm{p}<0.001)$ were substantially greater than the control for AV cases, while the overall ratio of thiol/thiol-disulfide to thiol-disulfide $(p<0.001)$ was much higher compared to the control group. Total thiol is a powerful antioxidant and total thiol and thiol-disulfide are regarded inflammatory initiators in the AV lesion. The thiol-thiol-disulfide total ratio may predict AV susceptibility to scar formation $(\mathrm{p}=0.002)$ and predict AV gravity by utilising the GAG scoring $(\mathrm{p}<0.001)$.

\section{References}

[1] D. D. Lynn, T. Umari, C. A. Dunnick, and R. P. Dellavalle, "The epidemiology of acne vulgaris in late adolescence," Adolesc. Health. Med. Ther., vol. 7,pp. 1-13, 2016.

[2] Ö. Karaaslan, Y. Hacimusalar, and M. E. Ceylan Bal, "Evaluation of thiol/disulfide homeostasis in patients with a first episode of major depressive disorder," reactions, vol. 11, pp. 13, 2019.

[3] A. Kükürt, V. Gelen, Ö. F. Başer, H. A. Deveci, and M. Karapehlivan, "Thiols: Role in oxidative stress-related disorders," in Lipid Peroxidation, IntechOpen, vol. 2, pp. 34-43, 2021.

[4] D. D. Demirseren, C. Cicek, M. Alisik, M. E. Demirseren, A. Aktaş, and O. Erel, "Dynamic thiol/disulphide homeostasis in patients with basal cell carcinoma," Cutan. Ocul. Toxicol., vol. 36, pp. 278-282, 2017.

[5] G. Kalkan, S. Emre, M. Alisik, A. Aktaş, and P. Baran, "Dynamic thiol/disulfide homeostasis in patients with lichen planus," J. Clin. Lab. Anal., vol. 33, pp. e22642, 2019.

[6] S. Emre, D. D. Demirseren, M. Alisik, A. Aktas, S. Neselioglu, and O. Erel, "Dynamic thiol/disulfide homeostasis and effects of smoking on homeostasis parameters in patients with psoriasis," Cutan. Ocul. Toxicol., vol. 36, pp. 393-396, 2017.

[7] S. D. Pektas, E. T. Alataş, G. Doğan, S. Neşelioğlu, and Ö. Erel, "A marker for evaluation of oxidative stress in patients with Alopecia Areta: Thiol-Disulphide Homeostasis," vol. 3, pp. 75-89, 2018.

[8] M. Aksoy and A. Kirmit, "Thiol/disulphide balance in patients with psoriasis," Adv. Dermatology Allergol. Dermatologii i Alergol., vol. 37,pp. 52, 2020.

[9] S. Sener, A. Akbas, F. Kilinc, P. Baran, O. Erel, and A. Aktas, "Thiol/disulfide homeostasis as a marker of oxidative stress in rosacea: a controlled spectrophotometric study," Cutan. Ocul. Toxicol., vol. 38, pp. 55-58, 2019.

[10] G. Gurel, M. Karadol, C. Bal, B. Iptec, and E. ÇÖLGEÇEN, "Evaluation of thiol/disulfide homeostasis in patients with acne vulgaris," vol. 3, pp. 1-13, 2019. 
[11]B. E. Prie, L. Iosif, I. Tivig, I. Stoian, and C. Giurcaneanu, "Oxidative stress in androgenetic alopecia,” J. Med. Life, vol. 9,pp. 79, 2016.

[12] İ. Sögüt, A. Ş. Aydın, E. S. Gökmen, P. G. Atak, Ö. Erel, and U. G. DeGrigo, "Evaluation of Oxidative Stress and Thiol-Disulfide Parameters According to the Body Mass Index in Adult Individuals.," Erciyes Med. Journal/Erciyes Tip Derg., vol. 40, 2018.
[13]A. Shroff, A. Mamalis, and J. Jagdeo, "Oxidative stress and skin fibrosis," Curr. Pathobiol. Rep., vol. 2, pp. 257-267, 2014.

[14] R. Vona, A. Giovannetti, L. Gambardella, W. Malorni, D. Pietraforte, and E. Straface, "Oxidative stress in the pathogenesis of systemic scleroderma: An overview," J. Cell. Mol. Med., vol. 22, pp. 3308-3314, 2018. 